\title{
Incidental use of ecstasy: no evidence for harmful effects on cognitive brain function in a prospective fMRI study
}

\author{
Gerry Jager • Maartje M. de Win • Hylke K. Vervaeke • \\ Thelma Schilt • Rene S. Kahn • Wim van den Brink • \\ Jan M. van Ree $\cdot$ Nick F. Ramsey
}

Received: 4 October 2006 / Accepted: 21 March 2007 /Published online: 3 May 2007

(C) Springer-Verlag 2007

\begin{abstract}
Rationale Heavy ecstasy use in humans has been associated with cognitive impairments and changes in cognitive brain function supposedly due to damage to the serotonin system. There is concern that even a single dose of 3,4methylenedioxymethamphetamine may be neurotoxic, but very little is known about the consequences of a low dose of ecstasy for cognitive brain function.

Objectives The objective of the study was to assess the effects of a low dose of ecstasy on human cognitive brain function using functional magnetic resonance imaging (fMRI). Materials and method We prospectively studied, as part of the NeXT (Netherlands XTC toxicity) study, sustained effects of a low dose of ecstasy on brain function in 25 subjects before and after their first episode of ecstasy use (mean 2.0 \pm 1.4 ecstasy pills, on average $11.1 \pm 12.9$ weeks since last ecstasy use), compared to 24 persistent ecstasy-naive controls, also
\end{abstract}

\section{R. S. Kahn}

Department of Psychiatry, A.01.126,

Rudolf Magnus Institute of Neuroscience,

University Medical Center Utrecht,

Heidelberglaan 100 ,

3584 CX Utrecht, The Netherlands

\section{M. de Win}

Department of Radiology,

University of Amsterdam,

Academic Medical Center Amsterdam,

Amsterdam, The Netherlands

H. K. Vervaeke

Bonger Institute of Criminology,

University of Amsterdam,

Amsterdam, The Netherlands

T. Schilt

Department of Psychiatry, University of Amsterdam,

Academic Medical Center Amsterdam,

Amsterdam, The Netherlands measured twice and matched with the novice users on age, gender, IQ, and cannabis use. Cognitive brain function was measured in the domains of working memory, selective attention, and associative memory using fMRI.

Results No significant effects were found of a low dose of ecstasy on working memory, selective attention, or associative memory neither at the behavioral level nor at the neurophysiological level.

Conclusions This study yielded no firm evidence for sustained effects of a low dose of ecstasy on human cognitive brain function. The present findings are relevant for the development of prevention and harm reduction strategies. Furthermore, the study is relevant to the discussion concerning potential therapeutic use of ecstasy.

Keywords Ecstasy $\cdot$ MDMA $\cdot$ Prospective . Functional MRI $\cdot$ Cognition $\cdot$ Brain function

W. van den Brink

Amsterdam Institute for Addiction Research and Department of Psychiatry, University of Amsterdam,

Academic Medical Center,

Amsterdam, The Netherlands

J. M. van Ree

Department of Pharmacology and Anatomy, Rudolf Magnus

Institute of Neuroscience, University Medical Center,

Utrecht, The Netherlands

N. F. Ramsey

Department of Neurosurgery, Rudolf Magnus Institute

of Neuroscience, University Medical Center Utrecht,

Utrecht, The Netherlands

G. Jager $(\bowtie)$

Department of Neurosurgery, A.01.126, Rudolf Magnus Institute

of Neuroscience, University Medical Center Utrecht,

Heidelberglaan 100,

3584 CX Utrecht, The Netherlands

e-mail: gjager@umcutrecht.nl 


\section{Introduction}

Ecstasy (3,4-methylenedioxymethamphetamine or MDMA) is a popular recreational drug. A large body of evidence indicates that MDMA has the potential to damage brain serotonin neurons in various animal species (Easton and Marsden 2006; Green et al. 2003). There is still controversy, however, whether similar serotonergic damage does occur in human ecstasy users (Gouzoulis-Mayfrank and Daumann 2006; Kish 2002). Nonetheless, ecstasy use has been frequently associated with a variety of functional sequelae, including psychological problems and cognitive impairments (Cole and Sumnall 2003; Parrott 2001). Functional neuroimaging studies in ecstasy users have reported changes in cognitive brain function as well. Both decreased and enhanced brain activity related to memory function has been observed in various brain regions, including frontal, temporal, visual, and limbic areas (Daumann et al. 2003, 2004, 2005; Moeller et al. 2004). However, some important questions remain unanswered. For one, most studies concern heavy ecstasy users, but there is concern that even a single dose of MDMA might be neurotoxic (Gijsman et al. 1999; McCann and Ricaurte 2001). For example, animal studies indicate that even after a single dose of MDMA, damage can occur in the serotonin system (Adori et al. 2006; O'Shea et al. 1998; Ricaurte et al. 1988). However, whether these animal findings can be extrapolated to humans is still debated (Easton and Marsden 2006). Second, interpretation of human data is hampered by the lack of baseline data (Gouzoulis-Mayfrank and Daumann 2006). Only a few prospective studies have been performed in ecstasy-naive volunteers, but these studies invariably focused on acute instead of sustained or long-term effects of ecstasy (Dumont and Verkes 2006). There are compelling reasons why more research is needed on the sustained effects of low dose ecstasy use in humans. First, the majority of recreational ecstasy users are incidental or moderate users (Rodgers et al. 2003; Scholey et al. 2004). Second, interest is growing in the possible beneficial actions of MDMA in posttraumatic stress disorder and in late stage cancer to reduce anxiety and agitation (MAPS Research Information ${ }^{1}$ ). Ideally, only a longitudinal prospective study in ecstasy-naive subjects randomly assigned to MDMA or placebo and conducted in a laboratory setting could answer the question whether ecstasy is neurotoxic in humans. However, given the potential neurotoxicity of MDMA, such a study is ethically not acceptable. Recently, it has been advocated to start longitudinal prospective studies in specific groups of young people who are at an increased risk for use of ecstasy

\footnotetext{
${ }^{1}$ MAPS Research Information at http://www.maps.org/mdma/ for information on approved phase I and II studies.
}

(Gouzoulis-Mayfrank and Daumann 2006). The current study is the first that succeeded in this approach. We investigated the effects of initial use of ecstasy on working memory, selective attention and associative memory, measured with functional magnetic resonance imaging (fMRI), using a prospective naturalistic design. Based on previous results with exactly the same fMRI task paradigms in heavy poly-substance ecstasy users which indicated poorer associative memory performance and altered brain activity in associative memory-related regions (Jager et al. 2007b), we hypothesized that first use of ecstasy would affect associative memory in terms of performance and of brain function, but not working memory and selective attention.

\section{Materials and methods}

This study is part of the Netherlands XTC Toxicity (NeXT) Study. A detailed description of the design and objectives of the NeXT study is provided in a paper on the methods (De Win et al. 2005). Besides fMRI, subjects underwent SPECT, MR imaging, and cognitive testing; results of these measurements are or will be reported in separate publications (De Win et al. 2007; Schilt et al. 2007).

\section{Subjects}

Between April 2002 and April 2004, a total of 188 young adults (18-35 years) were included in the prospective cohort study of the NeXT project. Of this cohort, 96 participated in the present fMRI study. At the time of inclusion, none of the subjects had ever used ecstasy, but they were selected for being at high risk of initiating the use of ecstasy in the near future (see below). Subject recruitment consisted of a combination of targeted site sampling, advertisement ${ }^{2}$, and snowball sampling referrals. For details on recruitment, inclusion and exclusion criteria, we refer to Vervaeke et al. (2006) and De Win et al. (2005). Main inclusion criterion was a high probability to start using ecstasy based on the intention to probably or certainly use ecstasy for the first time in the near future and/or ecstasy use by peers. All subjects were right-handed and were excluded if they reported: major medical or psychiatric disorders; current use of psychotropic medications; use of intravenous drugs; pregnancy; and contraindications for MRI. Except for smoking, which was allowed until $2 \mathrm{~h}$ before scanning, subjects had to abstain from psychoactive substances for at least 2 weeks and from alcohol for at least 1 week before testing. Compliance to abstinence was

\footnotetext{
${ }^{2}$ Advertisements were placed on the internet, on a special website for this study from the Academic Medical Center Amsterdam and by a pop-up advertising campaign on the Microsoft MSN Network.
} 
checked by urine drug screening (enzyme-multiplied immunoassay for amphetamines, ecstasy, opiates, cocaine, benzodiazepines, cannabis, and alcohol).

Subjects were fully informed about the potential risk of ecstasy use in the study information letter and gave their written consent according to the Helsinki Declaration. The local ethics committee approved the study. Subjects were paid for their participation ( 150 Euro per session of 2 days).

\section{Procedure}

At baseline, all 96 subjects underwent fMRI scanning and completed validated self-report questionnaires about their drug use (Van de Wijngaart et al. 1997). They were screened for axis I psychiatric disorders using the Dutch version of the mini international neuropsychiatric interview for DSM-IV clinical disorders (Sheehan et al. 1998). Urine samples were collected, and pre-morbid verbal intelligence was estimated using the Dutch version of the National Adult Reading Test (Schmand et al. 1991). After baseline examination, subjects were approached at regular intervals to fill out a questionnaire concerning drug use (four in total during a follow-up period of approximately 18 months). Within this follow-up period, 27 subjects started to use ecstasy. These subjects were invited for a second fMRI scan relatively soon after their first ecstasy use and with a maximum cumulative ecstasy dose of ten tablets. As a consequence of the latter criterion, one subject who has used 20 tablets had to be excluded from the current study, leaving 26 novice ecstasy users for the analysis. The control group, also scanned a second time, consisted of 24 subjects selected from the initial baseline sample who did not use ecstasy within the follow-up period, based on age, gender, IQ, and history of cannabis use (for individual matching with the users). Urine drug screening and drug use questionnaires were repeated.

Assessment of working memory, selective attention, and associative memory

Three fMRI tasks were administered: a working memory task based on Sternberg's item-recognition paradigm (denoted STERN), a visuo-auditory selective attention task (SAT), and a pictorial associative memory task (PMT) that depends on (para)hippocampal brain function.

The STERN task involves memorizing sets of five letters (the target set). After this, a series of ten letters is displayed in sequence (Fig. 1). Subjects have to decide whether subsequently presented letters belong to the set or not. Before scanning, subjects practiced on a specific set of letters for $21 \mathrm{~min}$. During scanning, two experimental tasks were administered, which differed only with regard to the target set(s): a novel set and a practiced set. In the practiced set task (PT), the specific set was used repeatedly. In the novel set task (NT), the composition of the target set was changed after every run of ten trials. In the scanner, both tasks were presented in eight epochs of ten stimuli each. An additional reaction time control task (CT) was included (also eight epochs of ten stimuli), as well as eight rest periods of equal epoch duration (for further details on the STERN, we refer to Jansma et al. 2001, 2004, and Ramsey et al. 2004).

The SAT is an oddball detection task (Fig. 2) and involves detection of deviant stimuli (either tones deviant in pitch from a baseline tone or dots deviant in size from a
Fig. 1 The temporal sequence of events is shown for the STERN task. Each epoch starts with presentation of the target set and is followed by ten trials. Subjects have to press a button as fast as possible if the letter belongs to the target set. Details are described in "Materials and methods"

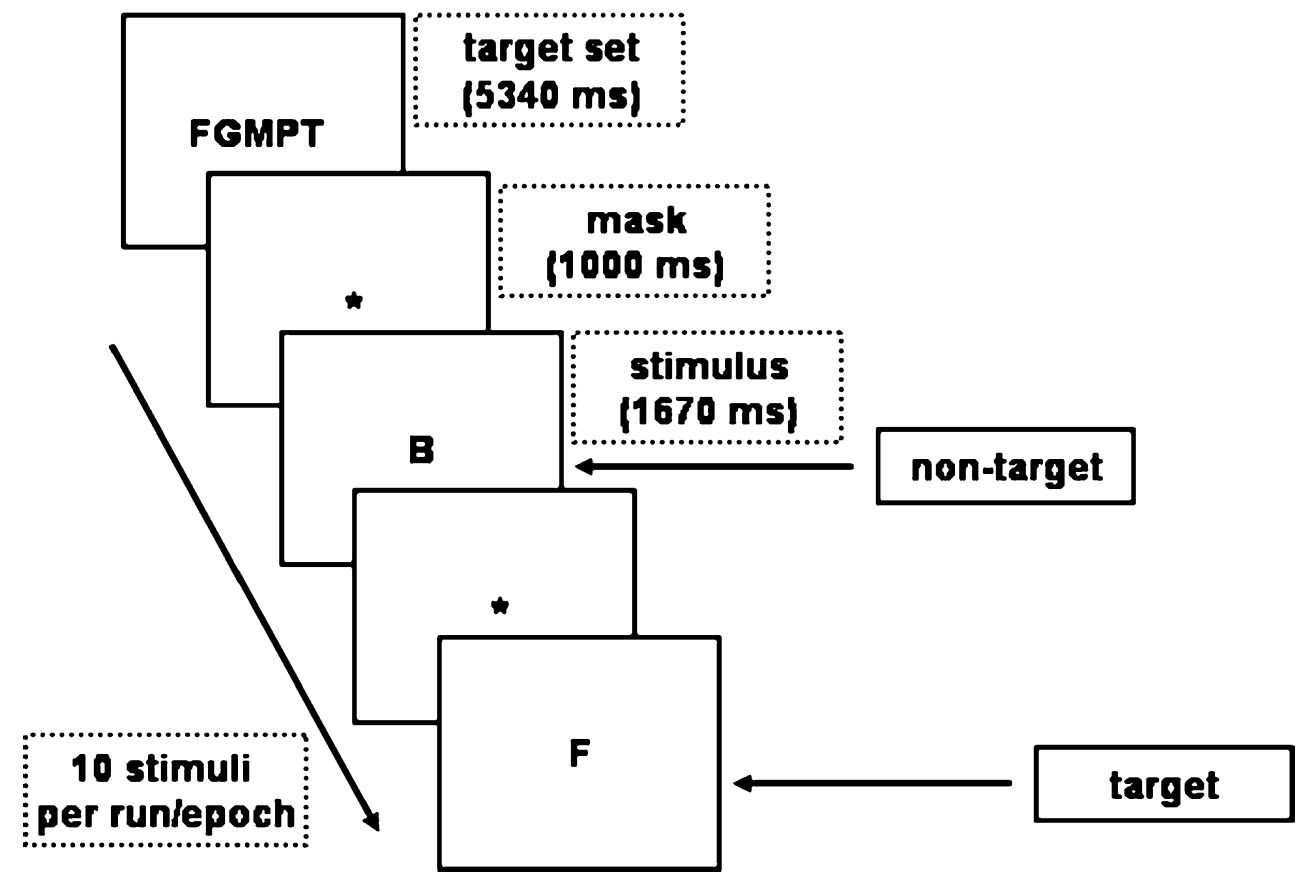


Fig. 2 The temporal sequence of events for the SAT task. Each epoch (duration 29 s) starts with an instruction slide (5 s), indicating 'rest', 'attend to tones only', or 'attend to dots only'. Both during 'tones only' and 'dots only', the instruction slide is followed by a series of 25 stimuli (simultaneous asynchronous presentation of tones and dots at a variable inter-stimulus interval rate) of which, on average, 20\% deviant (targets). In case of a target, subjects have to press a button as fast as possible. Before fMRI scanning, the difference between standard and deviant tones and dots is determined for each individual by changing the contrast until a performance of $80 \%$ correct is obtained

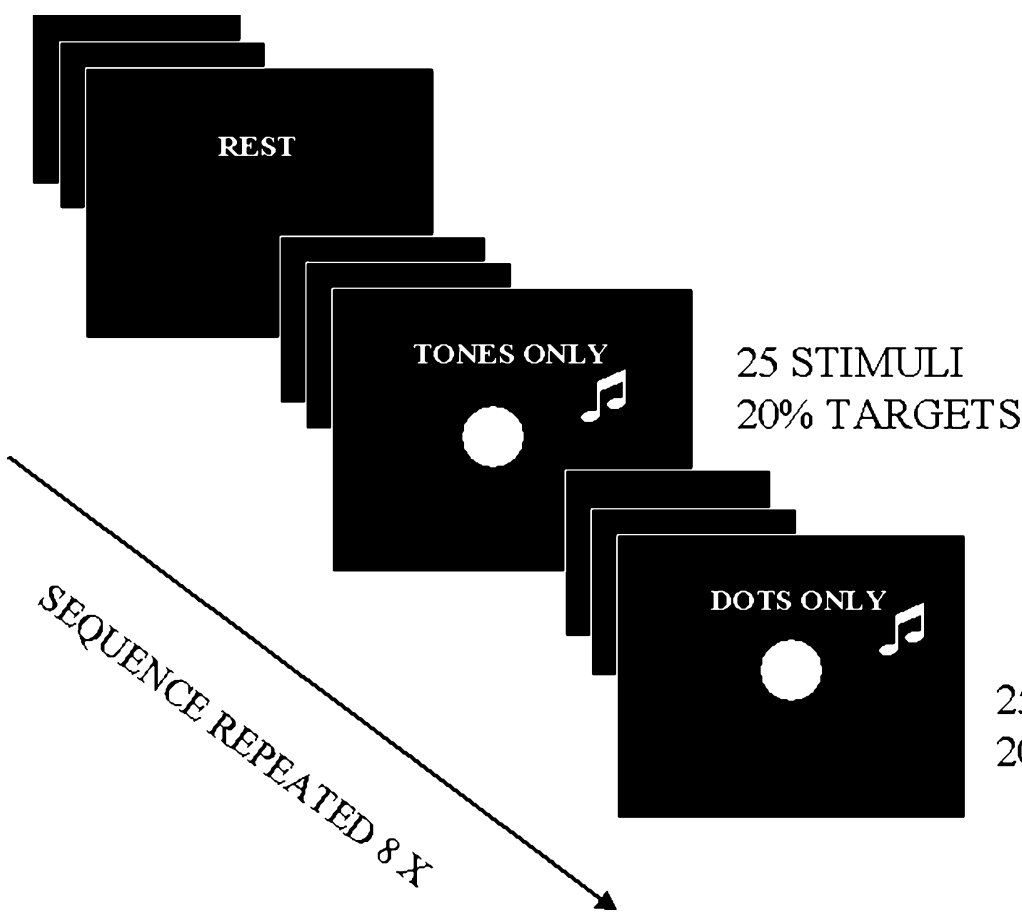

baseline dot). A threshold for detecting differences in pitch and dot size was determined individually before the scan session by adjusting it until the subject detected at least $80 \%$ of the deviant stimuli. Tones and dots were presented simultaneously with a variable interstimulus interval (mean $1.0 \mathrm{~s}$ ) and were presented in 16 epochs of $30 \mathrm{~s} \mathrm{each}$. At the start of each epoch, subjects were instructed to attend either to the tones while ignoring the dots (TO) or vice versa (DO). Eight rest periods (RS) of equal epoch duration were intermixed (for further details, we refer to Jager et al. 2006).
The PMT is a pictorial memory task modified from a paradigm from Henke et al. (1997). It involves three experimental tasks: (1) an associative learning task (AL) where subjects have to encode an association between two pictures; (2) a classification task in which single item pictures have to be classified (SC); and (3) a retrieval task (RE) where subjects have to recall specific picture pairs previously presented during the associative learning task. The RE task provides a performance measure. Figure 3 depicts a schematic example of the PMT task. Each picture
Fig. 3 The temporal sequence of events is shown for the PMT task. Each epoch starts with an instruction slide $(5 \mathrm{~s})$ followed by a fixation cross $(2.5 \mathrm{~s})$. This is followed by eight trials of $7.5 \mathrm{~s}$ each (picture pair $5 \mathrm{~s}$, fixation cross $2.5 \mathrm{~s}$ ). Subjects have to respond to the task by pressing one out of two buttons according to the instruction in each task condition. Details are described in "Materials and methods"

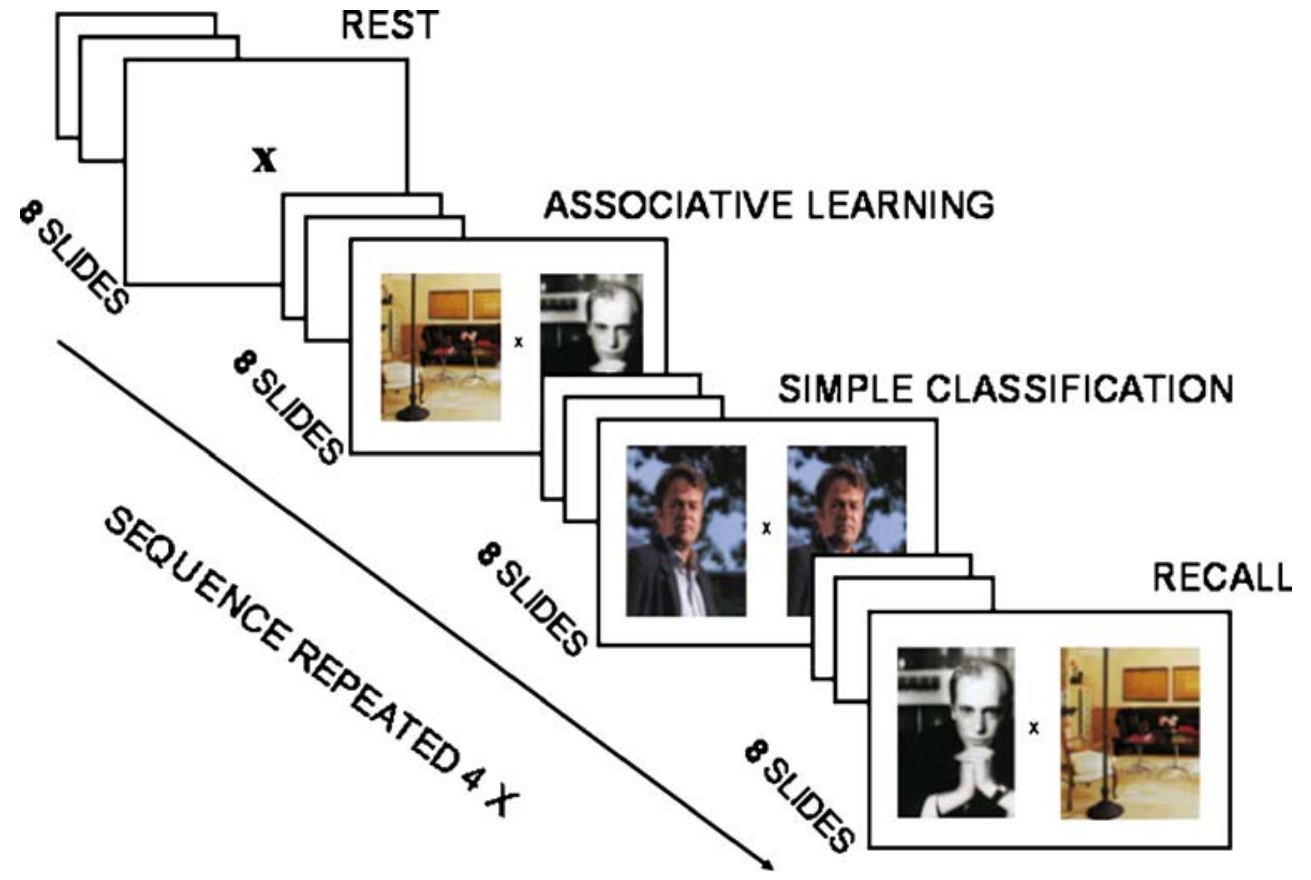


is presented for $5,000 \mathrm{~ms}$, followed by a 2,340-ms fixation cross. Each task is presented in four epochs (duration $65 \mathrm{~s}$ ) of eight stimuli. Also, four rest periods (RS) are included of equal epoch duration. A fixed-order sequence, i.e., RS, AL, $\mathrm{SC}$, and $\mathrm{RE}$, is repeated four times (for further details, we refer to Jager et al. 2007a).

\section{FMRI data acquisition}

Scans were made on a Philips ACS-NT 1.5 Tesla MRscanner with PT 6,000 gradients using a standard scan protocol (navigated 3D PRESTO; Ramsey et al. 1998). For the STERN task, a single run of 384 scans was acquired over a period of $18 \mathrm{~min}$ (scan parameters: TR $24.5 \mathrm{~ms}$, TE $35.5 \mathrm{~ms}$, flip angle $10.5^{\circ}$, FOV $208 \times 256 \times 120$, matrix $52 \times$ $64 \times 30$, voxel size $4.0 \mathrm{~mm}$ isotropic, scan time $2,800 \mathrm{~ms}$, 30 slices, transaxial orientation). Each epoch spanned 12 scans and lasted $33.6 \mathrm{~s}$. This was followed by one volume with a flip angle of $30^{\circ}$ for registration purposes. For the SAT task, a single run of 360 scans was acquired over a period of $12 \mathrm{~min}$. (scan parameters: TE $35.7 \mathrm{~ms}$ TR $23.7 \mathrm{~ms}$, flip angle $10.5^{\circ}$, FOV $208 \times 256 \times 88$, matrix $52 \times$ $64 \times 22$, voxel size $4.0 \mathrm{~mm}$ isotropic, time per scan 2,000 ms, 22 slices, transaxial orientation). Each epoch spanned 15 scans and lasted $30 \mathrm{~s}$. This was also followed by one volume with a flip angle of $30^{\circ}$ for registration purposes. Then, to allow for some rest in between tasks, a volumetric T1-weighted MR anatomical scan was acquired for spatial localization. Acquisition parameters were FOV $256 \times 256$, TR $30 \mathrm{~ms}$, TE $4.6 \mathrm{~ms}$, matrix $128 \times 128 \times 150$, flip angle $30^{\circ}$, with 150 slices of $1.2-\mathrm{mm}$ thickness. Finally, for PMT, a single run of 432 scans was acquired over a period of $18 \mathrm{~min}$ (scan parameters: TE $37 \mathrm{~ms}$ TR $24.4 \mathrm{~ms}$, flip angle $9^{\circ}$, FOV $208 \times 256 \times 104$, matrix $52 \times 64 \times 26$, voxel size $4.0 \mathrm{~mm}$ isotropic, time per scan $2,340 \mathrm{~ms}, 26$ slices, scan orientation parallel to the long axis of the hippocampus). Each epoch spanned 27 scans and lasted $63 \mathrm{~s}$. This was followed by one volume that was acquired with a flip angle of $30^{\circ}$ for registration purposes. Details on the scan procedure and scan parameters for STERN, SAT, and PMT have been previously described in Jansma et al. (2001), Ramsey et al. (2004), and Jager et al. (2007a).

Data and data analysis

\section{Demographic and drug use data}

Various aspects of ecstasy use were assessed [frequency of use, cumulative number of tablets, duration of use (months between first and last ecstasy use) and abstinence (number of weeks since last ecstasy use)]. Additional drug use data included lifetime and last year use of cannabis (number of joints), amphetamine and cocaine (number of occasions), and last year alcohol and tobacco consumption (drinks per week versus cigarettes per week). Demographic variables included age, gender, and verbal IQ.

\section{Performance data}

Outcome measures included performance accuracy (error rate for STERN, number correctly identified deviant stimuli for SAT, and percentage correct responses during RE for PMT) and reaction time (STERN only; measurements of mean reaction time during $\mathrm{CT}$, PT, and NT).

\section{fMRI data}

After reconstruction, functional and anatomical data were processed off-line using PV-wave ${ }^{\circledR}$ and Matlab ${ }^{\circledR}$ processing software. For all three tasks (STERN, SAT, and PMT), preprocessing of the fMRI data included several steps. First, after motion correction, individual statistical activity maps were generated for each of the task conditions compared to the rest condition by means of multiple regression analysis (Worsley and Friston 1995). Next, these maps were smoothed (FWHM $8 \mathrm{~mm}$ ) and normalized into standard MNI space (Collins et al. 1994). Then, to define the regions of interest (ROIs) for each task, the smoothed and normalized single-subject maps from the baseline session (when all subjects were ecstasy-naive) were combined into a group map, creating a contrast of interest for each task and using $z$ statistics (for details, see Jansma et al. 2001). For all contrasts, the statistical threshold for significant signal change was adjusted to yield separate regions of interest (ROIs), but always met the $p<0.05$ level, corrected for the total number of voxels in the brain. Using higher thresholds was necessary because with the large sample size $(n=49)$, ROIs merge when using the $p<0.05$ threshold. For STERN, the contrast NT-CT eliminated activity not directly involved in working memory and yielded several working memory-specific ROIs at a threshold of $z=6.0$ ( $p<0.01$, corrected) with a cluster size of at least 10 voxels. Using the same ROIs for all subjects, mean activity values (i.e., the average $z$ value of all voxels within the ROI) were obtained for CT, PT, and NT. ROIs were defined on the combined group map based on the baseline scans of all subjects. The same ROIs were used in the analyses of the fMRI data from the follow-up session after meticulous co-registration of the normalized follow-up functional scans onto the normalized baseline anatomy scan.

Similarly, for SAT, ROIs were defined based on the TORS contrast $z=6.0$ ( $p<0.01$, corrected, clusters $\geq 10$ voxels), and mean activity values were obtained for TO and DO.

For PMT, the contrast AL-SC eliminated activity not directly involved in associative learning and yielded several 
ROIs at a threshold of $z=5.0(p<0.01$, corrected, clusters $\geq 10$ voxels). For each of the ROIs, $\Delta t$ variables were computed, reflecting a measure for associative learning (contrasting $\mathrm{AL}$ with SC) and one for retrieval (contrasting RE with SC). $\Delta t$ variables were entered into statistical analyses.

The size of the (para)hippocampal ROIs was large, with activity extending into the fusiform gyrus. Because the (para)hippocampal regions are critically involved in associative memory processing, we added a second analysis to improve specificity by limiting the analysis to anatomically defined regions (right and left hippocampus, right and left parahippocampal gyrus). For this purpose, a segmentation procedure (Talairach and Tournoux 1988) was applied based on individual anatomical scans, yielding four separate clusters (right and left hippocampus, right and left parahippocampal gyrus) marked for each subject separately (for details, we refer to Jager et al. 2007a). Then, per individual, two statistical activity contrast maps based on an AL-SC and RE-SC contrast, respectively, $z=3.0 \quad(p<0.05$ corrected) were generated. After this, mean activity values within the four clusters for both contrast maps were obtained for each subject. Additionally, a voxel-by-voxel approach was applied within a confined search space, i.e., a small volume template was constructed of the (para) hippocampal region bilaterally (for details on this semi automatic small volume correction method, we refer to Matochik et al. 2003) in SPM2. Based on the smoothed single subject contrast maps group contrast maps were constructed. After this, group differences were tested using an $F$ test with a family-wise error correction (FWE) at $p<0.05$.

ROIs for STERN, SAT, and PMT are listed in Table 2. For each task and both scan sessions separately, mean activity values and $\Delta t$ variables were entered into GLM repeated measures analyses.

To check for group differences in activation outside the ROIs, the smoothed and normalized single subject maps were also combined into a group contrast map (data not shown) for each task, contrasting ecstasy-naive controls to incident ecstasy users using a voxel-by-voxel approach and $z$ statistics $(z=4.5, p<0.05$ corrected). Both during baseline, as well as during follow-up, the group contrast maps yielded no significant $(Z=4.5)$ differences in extent or magnitude of brain activity between the groups. This indicates that both novice ecstasy users and persistent ecstasy-naive controls yielded highly similar patterns of brain activity during baseline, and this was still the case during follow-up.

Finally, we compared the results from the aforementioned approach, i.e., using $t$ statistics in the first (fixed effects) and $z$ statistics in the second (random effects) stage of fMRI analyses, with the commonly used random-effects analysis for fMRI in SPM, i.e., entering first level regression-coefficients (or betas) into the second level random-effects group analysis. This yielded no significant outcome differences. Therefore, these data are not reported.

\section{Statistical analyses}

One-incident ecstasy user was excluded based on a positive urine test for cocaine, leaving 25 novice users for the analysis. Incidental technical malfunction of the MR scanner or the computer used for task presentation resulted in some missing or incomplete data. As a consequence, datasets were not complete for each of the included 49 subjects on each task paradigm, and results are reported for each task separately with the number of subjects that were included within brackets.

To test whether ecstasy users differed from ecstasy-naive controls at baseline and at follow-up in terms of age, verbal IQ and use of other substances than ecstasy (cannabis, amphetamine, cocaine, tobacco, and alcohol), $t$ tests and non-parametric Kolmogorov-Smirnov tests were applied.

To assess effects of ecstasy on task performance (accuracy, reaction times), GLM repeated measures analysis was applied for each task separately, with group as fixed factor and with session (baseline, follow-up) and task condition as within-subject factors.

Effects of ecstasy on brain activity were also tested using GLM repeated measures analysis, with brain activity as dependent variable and session, task condition, and ROIs (listed for all three tasks STERN, SAT, and PMT in Table 2) as within-subject factors.

Dose-response measures were examined using correlation analyses (Spearman's rho) in the group of novice ecstasy users with cumulative number of ecstasy tablets, duration of use, and duration of abstinence as predictor variables, and performance and brain activity as outcome variables. All analyses were performed using SPSS version 12.0.1 (SPSS, Chicago, IL, USA).

\section{Results}

Sample characteristics and drug use data

Table 1 shows baseline and follow-up characteristics on demographics and substance use of subjects that participated in the follow-up session. Incident ecstasy users used 2.0 tablets on average (range, 0.5-6, median 1.5 tablets) in a mean period of 6.7 weeks (SD 9.4) during an average follow-up period of 15.9 months (SD 4.6). The majority of novice ecstasy users had used ecstasy only once $(N=15 / 25$; $60 \%)$. At baseline, the two groups of future ecstasy users and persistent ecstasy-naive controls were similar in terms of gender distribution, age $(p=0.34)$, and verbal IQ $(p=$ 0.93). Also, the two groups did not differ in terms of 
Table 1 Demographics and characteristics of ecstasy use and use of other drugs (mean \pm SD)

\begin{tabular}{|c|c|c|c|c|}
\hline & \multicolumn{2}{|c|}{ Ecstasy users $(N=25)$} & \multicolumn{2}{|c|}{ Controls $(N=24)$} \\
\hline & Baseline & Follow-up & Baseline & Follow-up \\
\hline Gender & $9 \mathrm{M}, 16 \mathrm{~F}$ & & $8 \mathrm{M}, 16 \mathrm{~F}$ & \\
\hline $\mathrm{Age}^{\mathrm{a}, \mathrm{b}}$ & $21.8 \pm 2.5$ & $22.8 \pm 2.7$ & $21.2 \pm 1.8$ & $23.0 \pm 1.9$ \\
\hline DART-IQ ${ }^{\mathrm{a}, \mathrm{b}}$ & $103.4 \pm 7.4$ & & $103.6 \pm 10.0$ & \\
\hline \multicolumn{5}{|l|}{ Ecstasy } \\
\hline Cumulative dose (tablets) & & $2.0 \pm 1.4$ & & \\
\hline Time since last use (weeks) & & $11.1 \pm 12.9$ & & \\
\hline Duration of use (months) & & $1.2 \pm 2.4$ & & \\
\hline \multicolumn{5}{|l|}{ Other substances (last year) ${ }^{\mathrm{c}, \mathrm{d}}$} \\
\hline Alcohol (drinks/week) & $8.9 \pm 7.2$ & $9.1 \pm 7.6$ & $8.9 \pm 7.0$ & $7.2 \pm 5.5$ \\
\hline Tobacco (cigarettes/week) & $37.6 \pm 50.8$ & $23.8 \pm 37.6$ & $22.9 \pm 42.1$ & $22.5 \pm 35.4$ \\
\hline Cannabis (number of joints) & $25.1 \pm 38.5$ & $31.6 \pm 53.6$ & $19.8 \pm 31.9$ & $22.4 \pm 64.3$ \\
\hline Amphetamine (number of occasions) & $0.0 \pm 0.0$ & $0.0 \pm 0.0$ & $0.0 \pm 0.0$ & $0.0 \pm 0.0$ \\
\hline Cocaine (number of occasions) & $1.0 \pm 2.2$ & $1.4 \pm 2.6$ & $0.75 \pm 2.0$ & $0.25 \pm 1.2$ \\
\hline
\end{tabular}

smoking, use of alcohol, cannabis, amphetamines, and cocaine (all $p$ values $>0.50$ ). With the exception of ecstasy use, at follow-up, incident ecstasy users and controls were still very similar in terms of age and use of other drugs (all $p$ values $>0.40$ ).

\section{Performance data}

Performance data are summarized in Fig. 4.

\section{Working memory ( $N=44 ; 20$ incident ecstasy users, 24 persistent ecstasy-naive controls)}

Repeated measures analyses were performed on speed and accuracy of task performance as dependent variables, group (novice users, controls) as fixed factor, and session (baseline, follow-up) and task (CT, PT, and NT) as within-subject factors. These analyses yielded no significant group differences. Apart from a marginally significant three-way interaction for reaction time, between session, task and group $(F(1,41)=2.65, p<0.10)$, all other main or interaction effects of initial ecstasy use showed $p$ values $>0.30$.

Selective attention ( $N=43: 24$ incident ecstasy users, 19 persistent ecstasy-naive controls)

Repeated measures analysis revealed no significant effects of incident ecstasy use on threshold values for detecting $80 \%$ of the targets. Performance accuracy was similar for both groups (all $p$ values $>0.20$ ).
Associative memory ( $N=45 ; 24$ incident ecstasy users, 21 persistent ecstasy-naive controls)

Repeated measures analysis with performance accuracy as dependent variables, group as fixed factor, and session and task (SC, RE) as within-subject factors revealed no significant effects of group $(p>0.25)$, indicating that simple classification and recall accuracy were not affected by incident ecstasy use.

\section{Brain activity}

For STERN $(N=49)$, based on the NT-CT contrast, four ROIs reached significance in the frontal, parietal, and the fusiform gyrus. For SAT ( $N=47 ; 25$ users, 22 controls), based on the TO-RS contrast, six areas in the frontal, auditory, and visual cortex were activated above threshold. In addition, the TO-DO contrast yielded four areas in the inferior frontal, visual, and auditory cortices where brain activity was modulated by attention. Finally, for PMT $(N=49)$, the group contrast map (AL-SC) yielded nine ROIs in the (para)hippocampal regions bilaterally, the frontal and the occipital cortex. There was some overlap in ROIs between tasks. The anterior cingulate cortex was activated during all three tasks, whereas the right inferior frontal gyrus and the left dorsolateral prefrontal cortex were activated both during STERN and PMT. Table 2 shows ROIs, their MNI coordinates $(X, Y, Z)$, and peak activity values for each task. GLM repeated measures analyses of the fMRI signal in the ROIs were conducted for each task separately. 


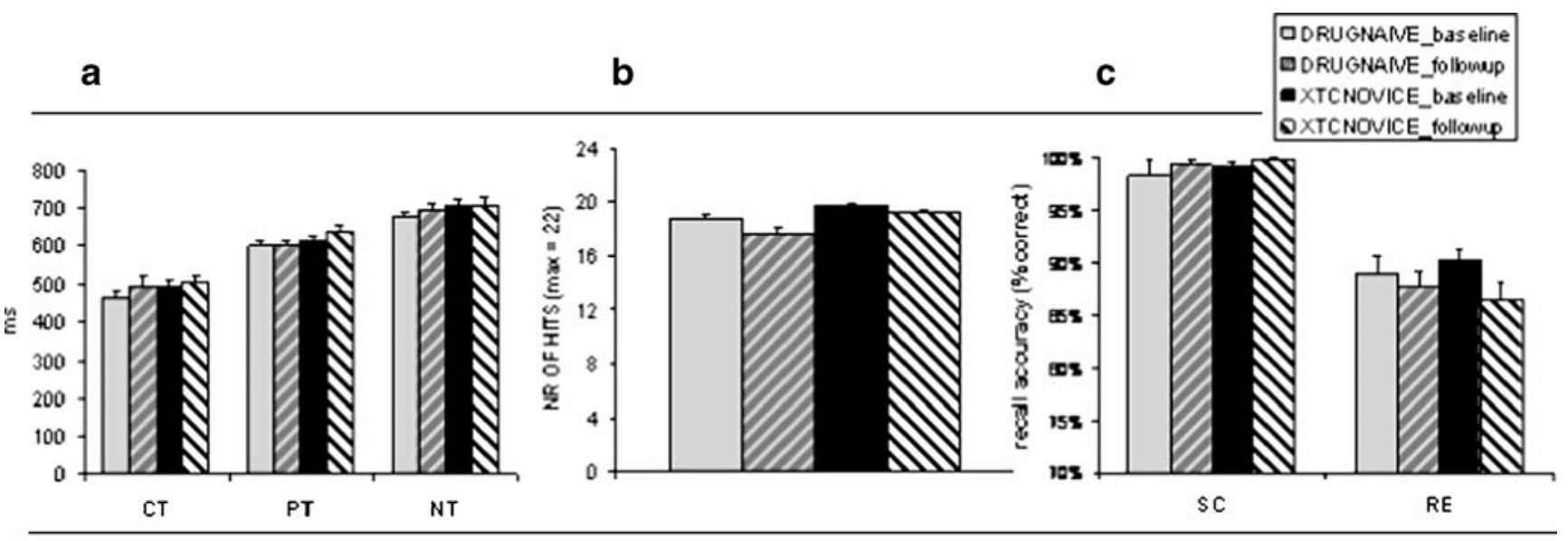

\section{STERN}

Fig. 4 Task performance on the STERN, SAT, and PMT during baseline and follow-up for initial ecstasy users and persistent ecstasynaive controls. a Reaction time (milliseconds) for reaction time control task $(C T)$, the practiced task $(P T)$, and the novel task $(N T)$. b

Working memory-related brain activity (STERN)

Whole brain analysis Two contrasts were examined. First, working memory activity (NT versus CT) was compared
SAT

\section{PMT}

Accuracy during SAT (number of correctly identified targets during tone and dot detection). c Accuracy during PMT (percentage correct answers) for the simple classification control task (SC) and the recognition task $(R E)$

between groups, with session and ROIs (Table 2) as within-subject factors. There were no significant differences between groups (all $p$ values $>0.20$ ), indicating that incident ecstasy use did not affect patterns of brain activity

Table 2 Brain regions of interest engaged in STERN, SAT, and PMT, respectively

\begin{tabular}{|c|c|c|c|c|c|c|c|}
\hline Task & Region & Brodmann area & Number of voxels & $X$ & $Y$ & $Z$ & Maximum $z$ value \\
\hline \multirow[t]{4}{*}{ STERN } & 1-SPC & 7 & 213 & 30 & -63 & 44 & 14.88 \\
\hline & 1-DLPFC & $9 / 46$ & 192 & 42 & 5 & 28 & 13.03 \\
\hline & $\mathrm{ACC}$ & $6 / 24$ & 73 & 6 & 9 & 52 & 13.82 \\
\hline & $1-\mathrm{FuG}$ & 37 & 64 & 46 & -63 & -12 & 10.03 \\
\hline \multirow[t]{6}{*}{ SAT } & r-IFG & 47 & 224 & -42 & 21 & 0 & 12.95 \\
\hline & 1-AUD & $41 / 42 / 22$ & 157 & 58 & -23 & 8 & 13.97 \\
\hline & $\mathrm{ACC}$ & $8 / 24$ & 130 & -2 & 21 & 44 & 15.41 \\
\hline & r-AUD & $41 / 42 / 22$ & 98 & -58 & -31 & 8 & 13.66 \\
\hline & $1-\mathrm{PcG}$ & 6 & 36 & 50 & -3 & 44 & 9.94 \\
\hline & 1-INS & 13 & 12 & 10 & 34 & 21 & 9.55 \\
\hline \multirow[t]{4}{*}{ SAT-attention ${ }^{\mathrm{a}}$} & VIS & 23 & 252 & -10 & -71 & 8 & 8.01 \\
\hline & 1-IFG & $9 / 47$ & 43 & 50 & 17 & 20 & 6.27 \\
\hline & 1-AUD & 42 & 33 & 60 & -31 & 8 & 5.51 \\
\hline & r-AUD & 42 & 23 & -58 & -27 & 0 & 6.13 \\
\hline \multirow[t]{9}{*}{ PMT } & r-PHG & $37 / 36$ & 153 & -26 & -47 & -12 & 13.05 \\
\hline & 1-PHG & $37 / 36$ & 134 & 26 & -47 & -12 & 12.29 \\
\hline & 1-DLPFC & 9 & 116 & 46 & 13 & 28 & 11.89 \\
\hline & 1-MOG & 19 & 98 & 34 & -87 & 16 & 10.01 \\
\hline & r-MOG & 18 & 95 & -34 & -87 & 12 & 10.76 \\
\hline & r-DLPFC & 46 & 41 & -50 & 29 & 20 & 7.37 \\
\hline & $\mathrm{ACC}$ & $8 / 24$ & 34 & 2 & 25 & 44 & 6.91 \\
\hline & r-IFG & 47 & 27 & -42 & 26 & -8 & 6.62 \\
\hline & 1-IFG & 47 & 22 & 50 & 21 & -8 & 6.54 \\
\hline
\end{tabular}

MNI coordinates are shown for the regions of interest involved in working memory (STERN), selective attention (SAT), and associative memory (PMT). The coordinates $X, Y$, and $Z$ represent location of the voxels with the highest $z$ value in the group map. Corresponding names and Brodmann areas are obtained from the location of voxels with the highest $z$ value.

l- Left, $r$-right, $S P C$ superior parietal cortex, $D L P F C$ dorsolateral prefrontal cortex, $A C C$ anterior cingulate cortex, $F u G$ fusiform gyrus, $I F G$ inferior frontal gyrus, $A U D$ auditory cortex, $P c G$ precentral gyrus, $I N S$ insula, $P H G$ parahippocampal gyrus, $M O G$ middle occipital gyrus, $V I S=$ visual cortex

${ }^{\text {a }}$ Shows the MNI coordinates for the regions where activity is modulated through attention during SAT. 
in the engaged network. Next, the effect of practice (NT versus PT, reflecting flexibility of the WM system; Jansma et al. 2001) was compared. This also revealed no significant differences between groups, indicating that ecstasy did not affect the way practice reduces activity in the WM network.

\section{Selective attention-related brain activity (SAT)}

Whole brain analysis Selective attention activity (based on the contrast TO versus RS) was compared between groups, with session and ROIs (Table 2) as within-subject factors. We found no significant effects of group (all $p$ values $>0.50$ ), indicating that initial ecstasy use did not affect patterns of brain activity in the involved network. Then, the modulating effect of attention on brain activity was compared between groups, with session and SAT-attention ROIs (Table 2) as within-subject factors. Again, no significant differences between groups were found (all $p$ values $>0.50$ ).

\section{Associative memory-related brain activity (PMT)}

Whole brain analysis Similar to STERN, for PMT, also two contrasts were examined. First, associative learning-related activity (AL versus SC) was compared between groups, with session and ROIs (Table 2) as within-subject factors. No significant differences between groups were found (all $p$ values $>0.50$ ). Second, brain activity related to recall (RE versus SC) was compared, also yielding no significant differences between groups (all $p$ values $>0.20$ ). This indicates that novice ecstasy use did not affect associative memory activity in the engaged network.

Region of interest analysis Next, analysis was restricted to four anatomically defined regions, i.e., right and left hippocampus and right and left parahippocampal gyrus. Again, two contrasts were examined: $\mathrm{AL}$ versus $\mathrm{SC}$ and $\mathrm{RE}$ versus SC, also not yielding significant differences between initial ecstasy users and persistent ecstasy-naive controls $(p$ values $>0.20$ ). In addition, a voxel-by-voxel approach was used in SPM2. At the FWE-corrected level of $p=0.05$, again, no differences were found between novice ecstasy users and ecstasy-naive controls.

Correlations between ecstasy use and brain activity

Within the group of novice ecstasy users $(N=25)$, we examined possible associations between two indices of ecstasy use, cumulative dose (total number of ecstasy tablets at follow-up) and weeks since last ecstasy use, and

Fig. 5 Regions of interest for STERN, SAT, and PMT: a Fusiform gyrus, b dorsolateral prefrontal cortex, c superior parietal cortex, $\mathbf{d}$ anterior cingulate cortex, $\mathbf{e}$ inferior frontal cortex, $\mathbf{f}$ auditory cortex, $\mathbf{g}$ pre-central gyrus, h (para)hippocampal region, i middle occipital gyrus. The numbers beneath the slices indicate the MNI $z$ coordinates. Slices are in radiological orientation (left side is right hemisphere and vice versa)

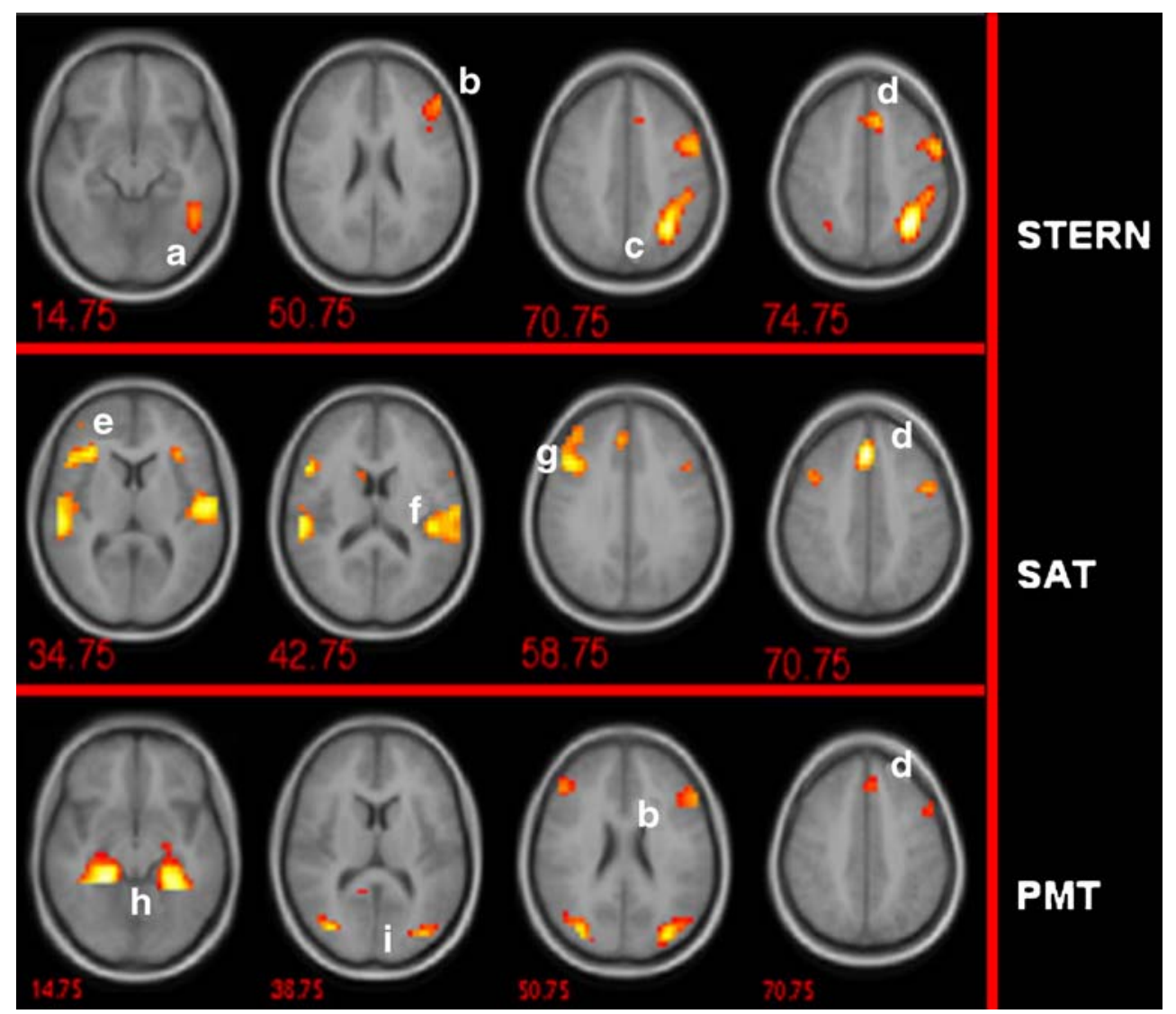


outcome variables (performance and brain activity) using Spearman's rho correlational analyses. To avoid type I errors, the level of significance was Bonferroni corrected, i.e., using a more stringent $p$ value adjusted for the number of ROIs. Duration of use was not included as an ecstasy use variable because the majority of subjects had used ecstasy only once $(N=15,60 \%)$, and thus, scored zero on this variable.

\section{Task performance}

No significant correlations were found between performance and cumulative dose or weeks since last use for all three tasks.

\section{Brain activity}

In line with the results on performance, we found no significant correlations between cumulative dose and weeks since last ecstasy use and brain activity in any of the regions of interest for STERN, SAT, and PMT (Fig. 5).

To examine whether the exclusion of one novice ecstasy user with a cumulative dose of 20 ecstasy tablets at follow-up did affect the results, all abovedescribed statistical analyses were re-run, this time including this particular subject. The main findings remained unchanged, as no significant effects of initial ecstasy use on performance and brain activity for all three tasks were found. There was only one tentative effect observed regarding the region of interest analysis for PMT. Including this subject with a cumulative dose of 20 tablets yielded a marginally significant interaction effect $[F(1,48)=2.80, p=0.10]$ between session and group. Ecstasy users tended to reveal slightly different patterns of overall brain activity in the four anatomical defined hippocampal and parahippocampal regions over sessions in comparison to the controls.

\section{Discussion}

The present fMRI study prospectively examined the nonacute effects of a single or low dose of ecstasy on cognitive brain function. We assessed task performance and brain activity patterns during working memory, selective attention, and associative memory in novice (but at least 2 weeks abstinent) ecstasy users before and after a period of first ecstasy use and compared these data with the same baseline and follow-up measurements of matched controls who did not use ecstasy during the follow-up period. We did not find firm evidence for sustained effects of initial ecstasy use on task performance in the domains of memory and attention. Also, no effect of incident ecstasy use was found on brain activity in the brain systems engaged in working memory, attention, or associative memory.

To date, this is the first prospective fMRI study on sustained effects of low-dose ecstasy use on cognitive brain function. Therefore, we can only compare our results with findings from studies investigating the (mainly acute) effects of MDMA on human neuropsychological function. In a recent review, Dumont and Verkes (2006) reported on all placebo-controlled studies that administered MDMA to healthy humans. Eleven tests in the attention domain were evaluated, but none of them had generated any significant effect. In the executive function domain, the literature search yielded only one study by Lamers et al. (2003), which reported no effect of MDMA on visual search, planning, or retrieval from semantic memory. Surprisingly, there are no studies about the acute effects of MDMA on memory, although cross-sectional studies into the long-term effects of ecstasy on human cognition most consistently report this domain to be impaired (Verbaten 2003).

We are aware of several limitations of the current study. For one, although prospective, the study design was naturalistic and not experimental. Therefore, there is uncertainty about dosage and purity of ecstasy tablets, and we had to rely on statements of the subjects themselves. However, because both novice users and persistent ecstasynaive controls were included for follow-up testing from the initial baseline cohort, we have no reason to question the truthfulness of their statements. Furthermore, pill testing confirms that in The Netherlands, more than $95 \%$ of the tablets sold as ecstasy contain MDMA as the sole (91.2\%) or main $(4.2 \%)$ psychoactive component (The Netherlands National Drug Monitor, 2004 ${ }^{3}$ ). Another limitation is that the sample was not randomly selected. Therefore, we cannot claim it to be statistically representative of the population of young people on the brink of experimenting with ecstasy. Yet, we do believe it is sufficiently varied, as we contacted candidates in many different places and ways. Moreover, in view of the specific demands of the study (a fairly demanding research project, including extensive brain imaging and blood sampling), even an initially random sample would have almost certainly reached a selective group in the end. In addition to sample selection, sample size would preferably have been larger, i.e., more subjects per group. Although difficult to realize, this would have further increased statistical power by reducing the chance of a type II error. Nevertheless, the number of subjects in the current study is larger than in other fMRI studies on effects of heavy ecstasy use, in which subjects numbers range between 6 and 15 ecstasy users and similar numbers of

\footnotetext{
${ }^{3}$ NDM 2004 at http://www.trimbos.n1/.
} 
controls, only examined once (for a review, see Cowan 2007). Thirdly, the pattern of use, the environmental circumstances during ecstasy consumption (ambient heat, dehydration), and the possible interaction with other substances, for instance alcohol, nicotine, cannabis, cocaine, or amphetamine (although use of the latter two was minimal), were not investigated. Therefore, we cannot exclude the possibility of confounding interactions between ecstasy and other drugs. More specifically, it has been suggested that cannabis attenuates the neurotoxic effects of ecstasy, as cannabis and ecstasy may have opposite effects on oxidative stress (Parrott et al. 2004). Ecstasy is thought to cause increased oxidative stress, thereby enhancing the risk for serotonergic neurotoxicity (Green et al. 2003), whereas cannabis may act as an antioxidant (Hampson et al. 2000), and therefore, possibly has neuroprotective effects (Sinor et al. 2000).

Finally, our failure to detect effects of a low dose of ecstasy on memory and attention and related brain function may be due to insufficient sensitivity and specificity of the task paradigms and the fMRI technique that was used. In this regard, it is important to note that in a previous study from our own laboratory, we did observe decreased memory performance and altered patterns of brain activity in various brain regions involved in associative memory in (poly-substance) frequent ecstasy users, applying exactly the same fMRI task paradigms and scanning procedures (Jager et al. 2007b). FMRI has been shown to be a sensitive tool in detecting neurocognitive impairments at the very early stage of multiple sclerosis (Audoin et al. 2006) in patients with various neuropsychiatric disorders, i.e., schizophrenia and obsessive-compulsive disorder (Jansma et al. 2004; Johnson et al. 2006; Van der Wee et al. 2003), and in substance users, i.e. cannabis users, tobacco smokers, and alcohol users (Bolla et al. 2005; Jacobsen et al. 2007; Myrick et al. 2004).

In conclusion, the present results provide no firm evidence for sustained effects of a low dose of ecstasy use on working memory, selective attention, and associative memory neither at the behavioral level nor at the neurophysiological level. The present findings are relevant for the development of prevention and harm reduction strategies, i.e., prevention of any drug use might be the preferred objective, but in many cases, harm reduction might be the only realistic option. It should be noted, however, that the result of a single prospective study using just one of the many available neurotoxicity detection techniques in a population with a rather narrow dose range is not sufficient for an evidence-based harm reduction strategy. Therefore, monitoring of the current sample is worthwhile because the expected increase in variation in dosage, frequency, and duration of ecstasy use within this group of novice users presents us with a unique opportunity for further research. In addition, more research with fMRI and other neuro- imaging techniques into the acute and sustained effects of MDMA on cognitive brain function is needed.

Acknowledgments This work was supported by a grant of The Netherlands Organization for Health Research and Development as part of their Addiction Program (ZonMW 310-00-036). We thank Microsoft MSN Network for their generous support concerning the internet pop-up advertisement campaign. We thank Dirk Korf for his valuable contribution to the design of the study, and finally, we thank Jeske Damoiseaux, Mieke Deenen, Judith Bosman, Rianne Petersen, Erika van Hell, and Lenny Ramsey for their assistance with data collection and analyses.

\section{References}

Adori C, Ando RD, Kovacs GG, Bagdy G (2006) Damage of serotonergic axons and immunolocalization of Hsp27, Hsp72, and Hsp90 molecular chaperones after a single dose of MDMA administration in Dark Agouti rat: temporal, spatial, and cellular patterns. J Comp Neurol 497:251-269

Audoin B, Van Au DM, Malikova I, Confort-Gouny S, Ibarrola D, Cozzone PJ, Pelletier J, Ranjeva JP (2006) Functional magnetic resonance imaging and cognition at the very early stage of MS. J Neurol Sci 245:87-91

Bolla KI, Eldreth DA, Matochik JA, Cadet JL (2005) Neural substrates of faulty decision-making in abstinent marijuana users. Neuroimage 26:480-492

Cole JC, Sumnall HR (2003) Altered states: the clinical effects of ecstasy. Pharmacol Ther 98:35-58

Collins DL, Neelin P, Peters TM, Evans AC (1994) Automatic 3D intersubject registration of MR volumetric data in standardized Talairach space. J Comput Assist Tomogr 18:192-205

Cowan RL (2007) Neuroimaging research in human MDMA users: a review. Psychopharmacology (Berl) 189:539-556

Daumann J, Schnitker R, Weidemann J, Schnell K, Thron A, GouzoulisMayfrank E (2003) Neural correlates of working memory in pure and polyvalent ecstasy (MDMA) users. Neuroreport 14:1983-1987

Daumann J Jr, Fischermann T, Heekeren K, Thron A, GouzoulisMayfrank E (2004) Neural mechanisms of working memory in ecstasy (MDMA) users who continue or discontinue ecstasy and amphetamine use: evidence from an 18-month longitudinal functional magnetic resonance imaging study. Biol Psychiatry 56:349-355

Daumann J, Fischermann T, Heekeren K, Henke K, Thron A, Gouzoulis-Mayfrank E (2005) Memory-related hippocampal dysfunction in poly-drug ecstasy (3,4-methylenedioxymethamphetamine) users. Psychopharmacology (Berl) 180:607-611

De Win MM, Jager G, Vervaeke HK, Schilt T, Reneman L, Booij J, Verhulst FC, Den Heeten GJ, Ramsey NF, Korf DJ, van den Brink W (2005) The Netherlands XTC Toxicity (NeXT) Study: objectives and methods of a study investigating causality, course, and clinical relevance. Int J Methods Psychiatr Res 14:167-185

De Win MM, Reneman L, Jager G, Vlieger EP, Olabarriaga SD, Lavini C, Bisschops I, Majoie CB, Booij J, den Heeten GJ, van den Brink W (2007) A prospective cohort study on sustained effects of low-dose ecstasy use on the brain in new ecstasy users. Neuropsychopharmacology 32:458-470

Dumont GJ, Verkes RJ (2006) A review of acute effects of 3,4methylenedioxymethamphetamine in healthy volunteers. J Psychopharmacol 20:176-187

Easton N, Marsden CA (2006) Ecstasy: are animal data consistent between species and can they translate to humans? J Psychopharmacol 20:194-210 
Gijsman HJ, Verkes RJ, Van Gerven JM, Cohen AF (1999) MDMA study. Neuropsychopharmacology 21:597

Gouzoulis-Mayfrank E, Daumann J (2006) Neurotoxicity of methylenedioxyamphetamines (MDMA; ecstasy) in humans: how strong is the evidence for persistent brain damage? Addiction 101:348-361

Green AR, Mechan AO, Elliott JM, O'Shea E, Colado MI (2003) The pharmacology and clinical pharmacology of 3,4-methylenedioxymethamphetamine (MDMA, "ecstasy"). Pharmacol Rev 55:463-508

Hampson AJ, Grimaldi M, Lolic M, Wink D, Rosenthal R, Axelrod J (2000) Neuroprotective antioxidants from marijuana. Ann N Y Acad Sci 899:274-282

Henke K, Buck A, Weber B, Wieser HG (1997) Human hippocampus establishes associations in memory. Hippocampus 7:249-256

Jacobsen LK, Pugh KR, Constable RT, Westerveld M, Mencl WE (2007) Functional correlates of verbal memory deficits emerging during nicotine withdrawal in abstinent adolescent cannabis users. Biol Psychiatry 61(1):31-40

Jager G, Kahn RS, van den Brink W, van Ree JM, Ramsey NF (2006) Long-term effects of frequent cannabis use on working memory and attention: an fMRI study. Psychopharmacology (Berl) 185:358-368

Jager G, van Hell HH, de Win MM, Kahn RS, van den Brink W, van Ree JM, Ramsey NF (2007a) Effects of frequent cannabis use on hippocampal activity during an associative memory task. Eur Neuropsychopharmacol 17:289-297

Jager G, de Win MM, van der Tweel I, Schilt T, Kahn RS, den Brink W, van Ree JM, Ramsey NF (2007b) Assessment of cognitive brain function in ecstasy users and contributions of other drugs of abuse: results from an fMRI study. Neuropsychopharmacology (Apr 25, in press)

Jansma JM, Ramsey NF, Slagter HA, Kahn RS (2001) Functional anatomical correlates of controlled and automatic processing. J Cogn Neurosci 13:730-743

Jansma JM, Ramsey NF, van der Wee NJ, Kahn RS (2004) Working memory capacity in schizophrenia: a parametric fMRI study. Schizophr Res 68:159-171

Johnson MR, Morris NA, Astur RS, Calhoun VD, Mathalon DH, Kiehl KA, Pearlson GD (2006) A functional magnetic resonance imaging study of working memory abnormalities in Schizophrenia. Biol Psychiatry 60(1):11-21

Kish SJ (2002) How strong is the evidence that brain serotonin neurons are damaged in human users of ecstasy? Pharmacol Biochem Behav 71:845-855

Lamers CT, Ramaekers JG, Muntjewerff ND, Sikkema KL, Samyn N, Read NL, Brookhuis KA, Riedel WJ (2003) Dissociable effects of a single dose of ecstasy (MDMA) on psychomotor skills and attentional performance. J Psychopharmacol 17:379-387

Matochik JA, London ED, Eldreth DA, Cadet JL, Bolla KJ (2003) Frontal cortical tissue composition in abstinent cocaine abusers: a magnetic resonance imaging study. Neuroimage 19:1095-1102

McCann UD, Ricaurte GA (2001) Caveat emptor: editors beware. Neuropsychopharmacology 24:333-336

Moeller FG, Steinberg JL, Dougherty DM, Narayana PA, Kramer LA, Renshaw PF (2004) Functional MRI study of working memory in MDMA users. Psychopharmacology (Berl) 177:185-194

Myrick H, Anton RF, Li X, Henderson S, Drobes D, Voronin K, George MS (2004) Differential brain activity in alcoholics and social drinkers to alcohol cues: relationship to craving. Neuropsychopharmacology 29:393-402

O'Shea E, Granados R, Esteban B, Colado MI, Green AR (1998) The relationship between the degree of neurodegeneration of rat brain 5-HT nerve terminals and the dose and frequency of administration of MDMA ('ecstasy'). Neuropharmacology 37:919-926
Parrott AC (2001) Human psychopharmacology of ecstasy (MDMA): a review of 15 years of empirical research. Hum Psychopharmacol 16:557-577

Parrott AC, Gouzoulis-Meyfrank E, Rodgers J, Solowij N (2004) Ecstasy/MDMA and cannabis: the complexities of their interactive neuropsychobiological effects. J Psychopharmacol 18:572-575

Ramsey NF, van den Brink JS, van Muiswinkel AM, Folkers PJ, Moonen CT, Jansma JM, Kahn RS (1998) Phase navigator correction in 3D fMRI improves detection of brain activation: quantitative assessment with a graded motor activation procedure. Neuroimage 8:240-248

Ramsey NF, Jansma JM, Jager G, Van Raalten T, Kahn RS (2004) Neurophysiological factors in human information processing capacity. Brain 127:517-525

Ricaurte GA, DeLanney LE, Irwin I, Langston JW (1988) Toxic effects of MDMA on central serotonergic neurons in the primate: importance of route and frequency of drug administration. Brain Res 446:165-168

Rodgers J, Buchanan T, Scholey AB, Heffernan TM, Ling J, Parrott AC (2003) Patterns of drug use and the influence of gender on self-reports of memory ability in ecstasy users: a web-based study. J Psychopharmacol 17:389-396

Schilt T, de Win MM, Koeter M, Jager G, Korf DJ, van den Brink W, Schmand B (2007) Cognition in novice Ecstasy users with minimal exposure to other drugs: a prospective cohort study. Arch Gen Psych (in press)

Schmand B, Bakker D, Saan R, Louman J (1991) [The Dutch reading test for adults: a measure of premorbid intelligence level]. Tijdschr Gerontol Geriatr 22:15-19

Scholey AB, Parrott AC, Buchanan T, Heffernan TM, Ling J, Rodgers $\mathrm{J}$ (2004) Increased intensity of ecstasy and polydrug usage in the more experienced recreational ecstasy/MDMA users: a WWW study. Addict Behav 29:743-752

Sheehan DV, Lecrubier Y, Sheehan KH, Amorim P, Janavs J, Weiller E, Hergueta T, Baker R, Dunbar GC (1998) The miniinternational neuropsychiatric interview (M.I.N.I.): the development and validation of a structured diagnostic psychiatric interview for DSM-IV and ICD-10. J Clin Psychiatry 59(Suppl 20):22-33

Sinor AD, Irvin SM, Greenberg DA (2000) Endocannabinoids protect cerebral cortical neurons from in vitro ischemia in rats. Neurosci Lett 278:157-160

Talairach J, Tournoux P (1988) Co-planar stereotaxic atlas of the human brain. Thieme, New York

Van de Wijngaart G, Braam R, de Bruin D, Fris M, Maalste NJM, Verbraec HT (1997) Ecstasy in het uitgaanscircuit (Ecstasy and the Dutch rave scene: a socio-epidemiologic study on the nature and extent of, and the risks involved in using ecstasy and other party drugs at dance events). Addiction Research Center Utrecht, The Netherlands

Van der Wee NJ, Ramsey NF, Jansma JM, Denys DA, van Megen HJ, Westenberg HM, Kahn RS (2003) Spatial working memory deficits in obsessive compulsive disorder are associated with excessive engagement of the medial frontal cortex. Neuroimage 20:2271-2280

Verbaten MN (2003) Specific memory deficits in ecstasy users? The results of a meta-analysis. Hum Psychopharmacol 18:281290

Vervaeke HK, Korf DJ, Benschop A, van den Brink W (2006) How to find future ecstasy users: targeted and snowball sampling in an ethically sensitive context. Addict Behav (Dec 22, in press)

Worsley KJ, Friston KJ (1995) Analysis of fMRI time-series revisitedagain. Neuroimage 2:173-181 